\title{
Structuring Acyclic Process Models
}

\author{
Artem Polyvyanyy $^{1}$, Luciano García-Bañuelos ${ }^{2}$, and Marlon Dumas ${ }^{2}$ \\ 1 Hasso Plattner Institute at the University of Potsdam, Germany \\ Artem.Polyvyanyy@hpi.uni-potsdam.de \\ ${ }^{2}$ Institute of Computer Science, University of Tartu, Estonia \\ \{luciano.garcia, marlon.dumas\}@ut.ee
}

\begin{abstract}
This paper addresses the problem of transforming a process model with an arbitrary topology into an equivalent well-structured process model. While this problem has received significant attention, there is still no full characterization of the class of unstructured process models that can be transformed into well-structured ones, nor an automated method to structure any process model that belongs to this class. This paper fills this gap in the context of acyclic process models. The paper defines a necessary and sufficient condition for an unstructured process model to have an equivalent structured model under fully concurrent bisimulation, as well as a complete structuring method.
\end{abstract}

\section{Introduction}

In the Business Process Modeling Notation (BPMN) and in similar notations, a process model is composed of nodes (e.g., tasks, events, gateways) connected by a "flow" relation. Although BPMN allows process models to have almost any topology, it is often preferable that process models follow some structure. In this respect, a well-known property of process models is that of (well-)structuredness [1], meaning that for every node with multiple outgoing arcs (a split) there is a corresponding node with multiple incoming arcs (a join), such that the set of nodes between the split and the join form a single-entry-single-exit (SESE) region. For example, Fig.1(a) shows an unstructured process model, while Fig.1(b) shows an equivalent structured model. Note that Fig.1(b) uses short-names for tasks (a, b, c ...), which appear next to each task in Fig.1(a).

This paper studies the problem of automatically transforming process models with arbitrary topology into equivalent well-structured models. The motivation for such a transformation is manifold. Firstly, it has been empirically shown that structured process models are easier to comprehend and less error-prone than unstructured ones [2]. Thus, a transformation from unstructured to structured process model can be used as a refactoring technique to increase process model understandability. Secondly, a number of existing process model analysis techniques only work for structured models. For example, a method for calculating cycle time and capacity requirements of structured process models is outlined in [3], while a method for analyzing time constraints in structured process models is presented in [4]. By transforming unstructured process models to structured ones, we can extend the applicability of these techniques to a larger class of models. Thirdly, a transformation from unstructured to structured process models can be 


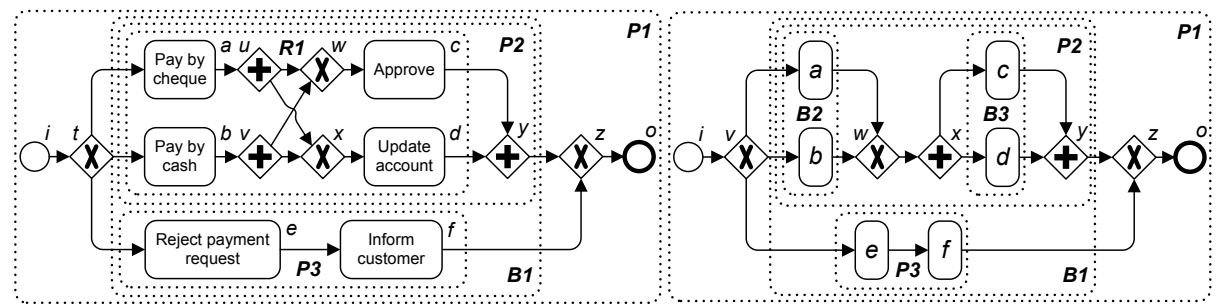

(a)

(b)

Fig. 1. Unstructured process model and its equivalent structured version

used to implement converters from graph-oriented process modeling languages to structured process modeling languages, e.g., BPMN-to-BPEL.

In the context of flowcharts, without parallel splits and joins, it has been shown that any unstructured flowchart can be transformed into a structured one [5]. If we add parallel splits and joins, this result no longer holds: There exist unstructured process models that do not have equivalent structured ones [1]. Several authors have attempted to classify the sources of unstructuredness in process models $[6,7,8]$ and to define automated methods for structuring process models $[9,10,11]$. However, these methods are incomplete: There is currently no full characterization of the class of inherently unstructured process models, i.e., unstructured process models that have no equivalent structured model. Also, none of the existing structuring methods is complete. In fact, this problem has not been fully solved even for acyclic process models. This paper fills this gap.

To streamline the presentation, we make several assumptions. Firstly, we consider process models composed of nodes (tasks, events, gateways) and control flow relations. In terms of BPMN, this means that we abstract away from other process model elements such as artifacts, annotations, associations, groups, pools, lanes, message flows, sub-process invocations and attributes associated to sub-process invocations, e.g., repetition. Nonetheless, the proposed method is applicable even if these types of elements are present in the input model. Simply, these ancillary elements and attributes need to be moved along with the tasks or events to which they are attached. In the same vein, we do not distinguish between events and tasks since, for the purpose of the transformation, both of these elements are treated equally. Secondly, we consider only sound process models [12]. This restriction is natural since soundness is a widely-accepted correctness criterion for process models. Thirdly, we consider process models in which every node has only one incoming or one outgoing arc. This restriction is merely syntactical because one can trivially split a node with multiple incoming and multiple outgoing arcs into two nodes: one node with a single outgoing arc and the other with a single incoming arc. Fourthly, we consider models with only one start node and one end node. Again, this is not a restriction since every sound model with multiple end nodes can be transformed into an equivalent sound model with a unique end node [12]. The reverse technique can be applied to models with multiple start nodes. Finally, we do not deal with the following BPMN constructs: OR gateways, complex gateways, error events and non-interrupting events. Lifting this latter restriction is left as future work. 
The next section presents a taxonomy of (unstructured) process components in process models and reviews related work. Next, Sect.3 introduces the formalism used to represent process models. Sect. 4 then introduces the behavioral equivalence used in this paper, viz. fully concurrent bisimulation (FCB), and shows that two acyclic process models are equivalent under this equivalence notion iff they have the same set of ordering relations. This result is used in Sect.5 to characterize the class of acyclic process components that can be structured and to define a structuring algorithm. Finally, Sect.6 concludes the paper.

\section{Background and Related Work}

This section discusses a complete taxonomy of process components. Next, we analyze previous work with respect to the proposed taxonomy.

\subsection{Taxonomy of Process Components}

The Refined Process Structure Tree (RPST) [13] is a technique to decompose a process model into a tree of regions. Each node in the RPST maps to a SESE region, herewith called a process component. A component in the RPST contains all components at the lower level, and all components at a given level are disjoint.

Each component in the RPST can be classified into one out of four classes [14]: A trivial $(T)$ component consists of a single flow arc. A polygon $(P)$ represents a sequence of components. A bond $(B)$ stands for a set of components that share two common nodes. Any other component is a rigid $(R)$. Rigid components explicitly define what makes a process model unstructured.

Fig.1 exemplifies the RPST decomposition in the form of dotted boxes. For instance in Fig.1(a), polygon $P 1$ is the root of the RPST and corresponds to the whole process model. Polygon $P 1$ contains bond $B 1$ that, in turn, contains polygons $P 2$ and P3. Observe that trivial components and polygons that are composed of two flow arcs are not visualized for simplicity reasons.

Trivials, polygons, and bonds are structured process components. If one could transform each rigid component in the RPST into an equivalent

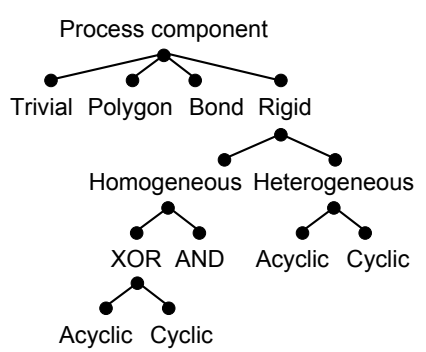

Fig. 2. Taxonomy structured component, the entire model could be structured by traversing the RPST bottom-up and replacing each rigid by its equivalent structured component. Accordingly, the rest of the paper focuses on structuring rigid components.

The methods for structuring rigid components differ depending on the types of gateways present in the rigid and whether the rigid contains cycles or not. We classify rigids as follows. A homogeneous rigid contains either only xor or only and gateways. We call these rigids (homogeneous) and rigids and (homogeneous) xor rigids, respectively. A heterogeneous rigid contains a mixture of and/xor gateways. Heterogeneous and homogeneous xor rigids are further classified into cyclic, if they contain at least one cycle, or acyclic. Importantly, a safe process model cannot contain homogeneous and rigids with cycles. Upon this background, a taxonomy of process components is provided in Fig.2. 


\subsection{Related Work}

The problem of structuring process models is relevant in the context of designing BPMN-to-BPEL transformations. However, BPMN-to-BPEL transformations such as [11] treat rigids as black-boxes that are translated using BPEL links or event handlers, rather than seeking to structure them. In this sense, the present contribution is complementary to this previous work.

A large body of work on flowcharts and GOTO program transformation [5], has addressed the problem of structuring xor rigids. In some cases, these transformations introduce additional boolean variables in order to encode part of the control flow, while in other cases they require certain nodes to be duplicated.

In [1], the authors show that not all acyclic and rigids can be structured. They do so by providing one counter-example, but do not give a full characterization of the class of models that can be structured nor do they define any automated transformation. Instead, they explore some causes of unstructuredness. In a similar vein, [6] presents a taxonomy of unstructuredness in process models, covering cyclic and acyclic rigids. But the taxonomy is incomplete, i.e., it does not cover all possible cases of models that can be structured. Also, the authors do not define an automated structuring algorithm.

In [7], the authors outline a classification of process components using region trees, a predecessor of the RPST. However, the authors do not provide a complete structuring method for acyclic heterogeneous rigids, e.g., the one in Fig.1(a). A similar remark applies to [10]. Meanwhile, [9] proposes a method for restructuring xor rigids based on GOTO program transformations, and extends this method to process graphs where such xor rigids are nested inside bonds. However, this method cannot deal with and rigids nor heterogeneous rigids.

\section{Preliminaries}

Below we introduce the notations used subsequently to represent process models.

\subsection{Petri Nets}

Petri nets are a well-known formalism for modeling concurrent systems. Below we present standard definitions of Petri nets and their semantics.

Definition 1 (Petri net). A Petri net, or a net, is a tuple $N=(P, T, F)$, with $P$ and $T$ as finite disjoint sets of places and transitions, and $F \subseteq(P \times T) \cup(T \times P)$ as the flow relation.

We identify $F$ with its characteristic function on the set $(P \times T) \cup(T \times P)$. We write $X=(P \cup T)$ for all nodes of a net. For a node $x \in X, \bullet x=\{y \in X \mid F(y, x)=1\}$ and $x \bullet=\{y \in X \mid F(x, y)=1\}$. A node $x \in X$ is an input (output) node of a node $y \in X$, iff $x \in \bullet y(x \in y \bullet)$. For $Y \subseteq X, \bullet Y=\bigcup_{y \in Y} \bullet y$ and $Y \bullet=\bigcup_{y \in Y} y \bullet$. We denote by $F^{+}$and $F^{*}$ irreflexive and, respectively, reflexive transitive closures of $F$.

Definition 2 (Net semantics). Let $N=(P, T, F)$ be a net.

○ $M: P \rightarrow \mathbb{N}_{0}$ is a marking of $N$, where $M(p), p \in P$, returns the number of tokens in place $p$. $[p]$ denotes the marking when place $p$ contains just one token and all other places contain no tokens. 
- For any transition $t \in T$ and for any marking $M$ of $N, t$ is enabled in $M$, denoted by $(N, M)[t\rangle$, iff $\forall p \in \bullet t: M(p) \geq 1$.

○ If $t \in T$ is enabled in $M$, then it can fire, which leads to a new marking $M^{\prime}$, denoted by $(N, M)[t\rangle\left(N, M^{\prime}\right)$. The new marking $M^{\prime}$ is defined by $M^{\prime}(p)=$ $M(p)-F(p, t)+F(t, p)$, for each place $p \in P$.

$\circ$ A sequence of transitions $\sigma=t_{1} \ldots t_{n}, n \in \mathbb{N}$, is a firing sequence, iff there exist markings $M_{0} \ldots M_{n}$, such that for all $1 \leq i \leq n$ holds $\left(N, M_{i-1}\right)\left[t_{i}\right\rangle\left(N, M_{i}\right)$.

- For any two markings $M$ and $M^{\prime}$ of $N, M^{\prime}$ is reachable from $M$ in $N$, denoted by $M^{\prime} \in[N, M\rangle$, iff there exists a firing sequence $\sigma$ leading from $M$ to $M^{\prime}$.

- A net system, or a system, is a pair $\left(N, M_{0}\right)$, where $N$ is a net and $M_{0}$ is a marking of $N . M_{0}$ is called the initial marking of $N$.

Workflow (WF-)nets [15] are a subclass of Petri nets specifically designed to represent business process models. A WF-net is a net with two special places: one to mark the start and the other the end of a workflow execution.

Definition 3 (WF-net, Short-circuit net, WF-system).

A Petri net $N=(P, T, F)$ is a workflow net, or a WF-net, iff $N$ has a dedicated source place $i \in P$, with $\bullet i=\varnothing, N$ has a dedicated sink place $o \in P$, with $o \bullet=\varnothing$, and the short-circuit net $N^{\prime}=\left(P, T \cup\left\{t^{\star}\right\}, F \cup\left\{\left(o, t^{\star}\right),\left(t^{\star}, i\right)\right\}\right), t^{\star} \notin T$, of $N$ is strongly connected. A $W F$-system is a pair $\left(N, M_{i}\right)$, where $M_{i}=[i]$.

Soundness and safeness are basic properties of WF-systems [15]. Soundness states that every execution of a WF-system ends with a token in the sink place, and once a token reaches the sink place, no other tokens remain in the net. Safeness refers to the fact that there is never more than one token in the same place.

In the rest of the paper, we also use three structural subclasses of Petri nets (free-choice net, occurrence net, and causal net), as well as labeled Petri nets to distinguish observable and silent transitions. These are defined below.

Definition 4 (Free-choice net, Occurrence net, Causal net). A Petri net $N=(P, T, F)$ is a free-choice net, iff $\forall p \in P,|p \bullet|>1: \bullet(p \bullet)=\{p\}$. Let $N=(P, T, F)$ be a net such that $\forall x, y \in P \cup T:(x, y) \in F^{+} \Rightarrow(y, x) \notin F^{+}$.

$\circ$ Net $N$ is an occurrence net, iff $\forall p \in P:|\bullet p| \leq 1$.

$\circ$ Net $N$ is a causal net, iff $\forall p \in P:|\bullet p| \leq 1 \wedge|p \bullet| \leq 1$.

Definition 5 (Labeled net). A labeled net is a tuple $N=(P, T, F, \mathcal{T}, \lambda)$, where $(P, T, F)$ is a net, $\mathcal{T}$ is a set of labels, such that $\tau \in \mathcal{T}$, and $\lambda: T \rightarrow \mathcal{T}$ is a function that assigns labels to transitions. If $\lambda(t) \neq \tau$, then $t$ is observable; otherwise, $t$ is silent. $\lambda$ is distinctive if it is injective on a subset of observable transitions.

\subsection{Process Model}

As discussed in Sect.1, we consider process models consisting of activities and gateways, as captured in the following definition.

\section{Definition 6 (Process model).}

A process model is a tuple $W=\left(A, G^{+}, G^{\times}, C, \mathcal{A}, \mu\right)$, where $A$ is a non-empty set of activities (or tasks), $G^{+}$is a set of and gateways, $G^{\times}$is a set of xor gateways (these sets are disjoint). We write $G=\left(G^{+} \cup G^{\times}\right)$for all gateways and $Z=(A \cup G)$ for all nodes of a model. $C \subseteq Z \times Z$ defines the control flow. $\mathcal{A}$ is a non-empty set of names and $\mu: A \rightarrow \mathcal{A}$ is a function that assigns names to tasks. 


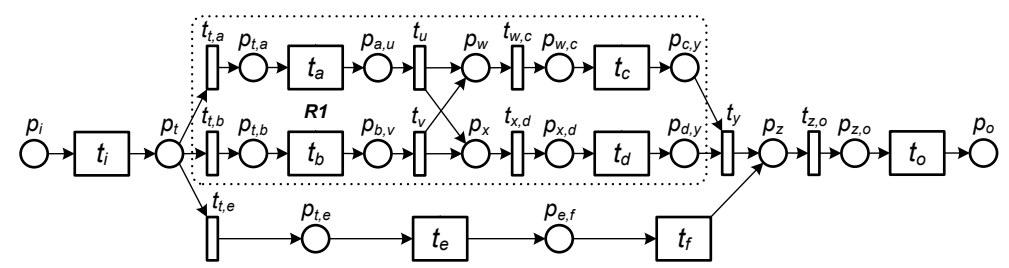

Fig. 3. A WF-net that corresponds to the process model in Fig.1(a)

A task $a \in A$ is a source, iff $\bullet a=\varnothing$ and it is a sink, iff $a \bullet=\varnothing$, where $\bullet x, x \in Z$, stands for a set of immediate predecessors and $x \bullet$ stands for a set of immediate successors of node $x$. As discussed in Sect.1, we assume that $(Z, C)$ is a graph with a single source, a single sink and is such that every node is on a path from the source to the sink. Each task $a \in A$ has at most one incoming and at most one outgoing arc, i.e., $|\bullet a| \leq 1 \wedge|a \bullet| \leq 1$, while each gateway is either a split or a join: A gateway $g \in G$ is a split, iff $|\bullet g|=1 \wedge|g \bullet|>1$. A gateway $g \in G$ is a join, iff $|\bullet g|>1 \wedge|g \bullet|=1$. The execution semantics of process models is defined by a mapping to labeled free-choice Petri nets (cf. Definition 7). For example, Fig.3 shows the WF-net of the process model in Fig.1(a). The figure highlights the subnet that corresponds to rigid component $R 1$ in Fig.1(a) (cf. dotted box).

Definition 7 (WF-net of a process model). Let $W=\left(A, G^{+}, G^{\times}, C, \mathcal{A}, \mu\right)$ be a process model. Let $I$ and $O$ be sources and sinks of $W$, respectively. The labeled net $N=(P, T, F, \mathcal{T}, \lambda)$ corresponding to $W$ is defined by:

○ $P=\left\{p_{x} \mid x \in G^{\times}\right\} \cup\left\{p_{x, y} \mid(x, y) \in C \wedge y \in A \cup G^{+}\right\} \cup\left\{p_{x} \mid x \in I \cup O\right\}$.

○ $T=\left\{t_{x} \mid x \in A \cup G^{+}\right\} \cup\left\{t_{x, y} \mid(x, y) \in C \wedge x \in G^{\times}\right\}$.

$\circ F=\left\{\left(t_{x}, p_{y}\right) \mid(x, y) \in C \wedge x \in A \cup G^{+} \wedge y \in G^{\times}\right\} \cup\left\{\left(t_{x}, p_{x, y}\right) \mid(x, y) \in C \wedge x, y \in\right.$

$\left.A \cup G^{+}\right\} \cup\left\{\left(t_{x, y}, p_{y}\right) \mid(x, y) \in C \wedge x, y \in G^{\times}\right\} \cup\left\{\left(t_{x, y}, p_{x, y}\right) \mid(x, y) \in C \wedge x \in\right.$

$\left.G^{\times} \wedge y \in A \cup G^{+}\right\} \cup\left\{\left(p_{x}, t_{x, y}\right) \mid(x, y) \in C \wedge x \in G^{\times}\right\} \cup\left\{\left(p_{x, y}, t_{y}\right) \mid(x, y) \in C \wedge y \in\right.$ $\left.A \cup G^{+}\right\} \cup\left\{\left(p_{x}, t_{x}\right) \mid x \in I\right\} \cup\left\{\left(t_{x}, p_{x}\right) \mid x \in O\right\}$.

○ $\mathcal{T}=\mathcal{A} \cup\{\tau\} . \lambda\left(t_{x}\right)=\mu(x), t_{x} \in T, x \in A$, otherwise $\lambda(t)=\tau, t \in T$.

Definition 7 states that a task is mapped to a Petri net transition with a single input and a single output arc. An and gateway maps to a transition with multiple outgoing arcs (and-split) or multiple incoming arcs (and-join). An xor gateway maps to a place with multiple outgoing arcs (xor-split) or multiple incoming arcs (xor-join). The places corresponding to xor-splits are immediately followed by empty $(\tau)$ transitions representing the branching conditions (cf. transitions $t_{x, y}$ introduced in the mapping). Sources and sinks of the process model are mapped to places.

A process model is sound if its corresponding WF-net is sound. In this paper we only consider sound process models. We note that a sound free-choice WFsystem is guaranteed to be safe [16] and Definition 7 always produces free-choice WF-nets. Thus the rest of the paper deals with sound and safe process models.

\section{Behavioral Equivalence of Process Models}

This section motivates fully concurrent bisimulation as the equivalence notion for process models, cf., Sect.4.1, and discusses the procedure of checking equivalence for the class of behavior captured by occurrence nets, cf., Sect.4.2. 


\subsection{Fully Concurrent Bisimulation}

An unstructured model and the corresponding structured model are structurally different, but behaviorally equivalent. There exist many notions of behavioral equivalence for concurrent systems [17]. A common notion of behavioral equivalence for concurrent systems is that of bisimulation. Related notions are those of weak bisimulation and branching bisimulation, which abstract away from silent transitions. These notions have been advocated as being suitable for comparing process models [12]. However, we argue that they are not suitable for our purposes. These three notions adopt an interleaving semantics - i.e., no two tasks are executed exactly at the same time. Thus, a concurrent system and its se-

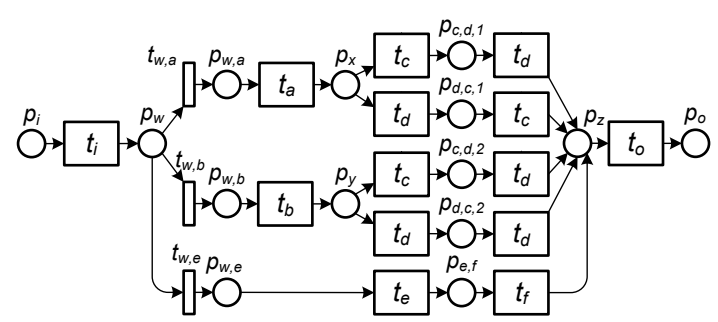
quential simulation are considered equivalent. For example, Fig. 4 shows the sequential simulation of the net in Fig.3. This net is structured and weakly bisimilar to the net in Fig.3, but it contains no parallel branch. We could take any process model, compute its sequential simulation, structure this sequential net using GOTO program transformations, and transform back the resulting sequential net into a structured process model. This structuring method is complete, but if we start with a process model containing and gateways, we obtain a (much larger) structured process model without any parallel branches.

Accordingly, we adopt a notion of equivalence that preserves the level of concurrency of observable transitions, viz. fully concurrent bisimulation (FCB) [18]. FCB is defined in terms of concurrent runs of a system, a.k.a. processes in the literature (but not to be confused with "business processes" or workflows).

Let $N=(P, T, F)$ be a causal net. A $P$-cut $c \subseteq P$ of $N$ is a maximal set of places unordered w.r.t. $F^{+}$. Let $\operatorname{Min}(N)$ define the set $\{x \in X \mid \bullet x=\varnothing\}$ and let $\operatorname{Max}(N)$ define the set $\{x \in X \mid x \bullet=\varnothing\}$.

Definition 8 (Process). A process $\pi=\left(N_{\pi}, \rho\right)$ of a system $S=\left(N, M_{0}\right), N=$ $(P, T, F)$, consists of a causal net $N_{\pi}=\left(P_{\pi}, T_{\pi}, F_{\pi}\right)$ and a function $\rho: X_{\pi} \rightarrow X$ : ○ $\rho\left(P_{\pi}\right) \subseteq P, \rho\left(T_{\pi}\right) \subseteq T$,

- $\operatorname{Min}\left(N_{\pi}\right)$ is a P-cut, which corresponds to the initial marking $M_{0}$, that is $\forall p \in P: M_{0}(p)=\left|\rho^{-1}(p) \cap \operatorname{Min}\left(N_{\pi}\right)\right|$, and

$\circ \forall t \in T_{\pi} \forall p \in P:\left(F(p, \rho(t))=\left|\rho^{-1}(p) \cap \bullet t\right|\right) \wedge\left(F(\rho(t), p)=\left|\rho^{-1}(p) \cap t \bullet\right|\right)$. A process $\pi$ of $S$ is initial, iff $T_{\pi}=\varnothing$.

A process $\pi^{\prime}$ is an extension of a process $\pi$ if it is possible to observe $\pi$ before one observes $\pi^{\prime}$. Consequently, process $\pi$ is a prefix of $\pi^{\prime}$.

\section{Definition 9 (Prefix, Process extension).}

Let $\pi=\left(N_{\pi}, \rho\right), N_{\pi}=\left(P_{\pi}, T_{\pi}, F_{\pi}\right)$, be a process of $S=\left(N, M_{0}\right), N=(P, T, F)$. Let $c$ be a P-cut of $N_{\pi}$ and let $c^{\downarrow}$ be the set $\left\{x \in X_{\pi} \mid \exists y \in c:(x, y) \in F^{*}\right\}$. A process $\pi_{c}^{\downarrow}$ is a prefix of $\pi$, iff $\pi_{c}^{\downarrow}=\left(\left(P_{\pi} \cap c^{\downarrow}, T_{\pi} \cap c^{\downarrow}, F \cap\left(c^{\downarrow} \times c^{\downarrow}\right)\right),\left.\rho\right|_{c^{\downarrow}}\right)$. A process $\pi^{\prime}$ is an extension of process $\pi$ if $\pi$ is a prefix of $\pi^{\prime}$. 
In order to define FCB, we need two auxiliary definitions: $\lambda$-abstraction of a process, which is a process footprint that ignores silent transitions, and the order-isomorphism of $\lambda$-abstractions.

Definition 10 (Abstraction of a process of a labeled system).

Let $S=\left(N, M_{0}\right), N=(P, T, F, \mathcal{T}, \lambda)$, be a labeled system and let $\pi=\left(N_{\pi}, \rho\right)$, $N_{\pi}=\left(P_{\pi}, T_{\pi}, F_{\pi}\right)$, be a process of $S$. The $\lambda$-abstraction of $\pi$, denoted by $\alpha_{\lambda}(\pi)=\left(T_{\pi}^{\prime},<, \lambda^{\prime}\right)$, is defined by $T_{\pi}^{\prime}=\left\{t \in T_{\pi} \mid \lambda(\rho(t)) \neq \tau\right\},<=\left\{\left(t_{1}, t_{2}\right) \in\right.$ $\left.T_{\pi}^{\prime} \times T_{\pi}^{\prime} \mid\left(t_{1}, t_{2}\right) \in F^{+}\right\}$, and $\lambda^{\prime}: T_{\pi}^{\prime} \rightarrow \mathcal{T}$, such that $\lambda^{\prime}(t)=\lambda(\rho(t)), t \in T_{\pi}^{\prime}$.

Two $\lambda$-abstractions are order-isomorphic if there exists a one-to-one correspondence between transitions of both abstractions that also preserves the ordering of the corresponding transitions in the respective abstractions.

Definition 11 (Order-isomorphism of abstractions).

Let $\alpha_{\lambda_{1}}=\left(T_{1},<_{1}, \lambda_{1}\right)$ and $\alpha_{\lambda_{2}}=\left(T_{2},<_{2}, \lambda_{2}\right)$ be two $\lambda$-abstractions, both with labels in $\mathcal{T}$. Then $\alpha_{\lambda_{1}}$ and $\alpha_{\lambda_{2}}$ are order-isomorphic, denoted by $\alpha_{\lambda_{1}} \cong \alpha_{\lambda_{2}}$, iff there is a bijection $\beta: T_{1} \rightarrow T_{2}$ such that $\forall t \in T_{1}: \lambda_{1}(t)=\lambda_{2}(\beta(t))$ and $\forall t_{1}, t_{2} \in T_{1}: t_{1} \prec_{1} t_{2} \Leftrightarrow \beta\left(t_{1}\right) \prec_{2} \beta\left(t_{2}\right)$.

Given the above, fully concurrent bisimulation is defined as follows.

Definition 12 (Fully concurrent bisimulation).

Let $S_{1}=\left(N_{1}, M_{0}^{1}\right)$ and $S_{2}=\left(N_{2}, M_{0}^{2}\right)$ be labeled systems, $N_{1}=\left(P_{1}, T_{1}, F_{1}, \mathcal{T}_{1}, \lambda_{1}\right)$ and $N_{2}=\left(P_{2}, T_{2}, F_{2}, \mathcal{T}_{2}, \lambda_{2}\right) . S_{1}$ and $S_{2}$ are fully concurrent bisimilar, denoted by $S_{1} \approx S_{2}$, iff there is a set $\mathcal{B} \subseteq\left\{\left(\pi_{1}, \pi_{2}, \beta\right)\right\}$, such that:

(i) $\pi_{1}$ is a process of $S_{1}, \pi_{2}$ is a process of $S_{2}$, and $\beta$ is a relation between the non- $\tau$ transitions of $\pi_{1}$ and $\pi_{2}$.

(ii) If $\pi_{0}^{1}$ and $\pi_{0}^{2}$ are the initial processes of $S_{1}$ and $S_{2}$, respectively, then $\left(\pi_{0}^{1}, \pi_{0}^{2}, \varnothing\right) \in \mathcal{B}$

(iii) If $\left(\pi_{1}, \pi_{2}, \beta\right) \in \mathcal{B}$, then $\beta$ is an order-isomorphism between the $\lambda_{1}$-abstraction of $\pi_{1}$ and the $\lambda_{2}$-abstraction of $\pi_{2}$.

(iv) $\forall\left(\pi_{1}, \pi_{2}, \beta\right) \in \mathcal{B}$ :

(a) If $\pi_{1}^{\prime}$ is an extension of $\pi_{1}$, then $\exists\left(\pi_{1}^{\prime}, \pi_{2}^{\prime}, \beta^{\prime}\right) \in \mathcal{B}$ where $\pi_{2}^{\prime}$ is an extension of $\pi_{2}$ and $\beta \subseteq \beta^{\prime}$.

(b) Vice versa.

FCB defines an equivalence relation on labeled systems that is stricter than weak bisimulation and related notions. The nets in Fig.4 and Fig. 3 are weakly bisimilar but not FCB-equivalent. Meanwhile, the two models in Fig.1 are FCB-equivalent (with the understanding that two process models are FCB-equivalent if the corresponding Petri nets are FCB-equivalent).

\subsection{Behavioral Equivalence and Ordering Relations}

The above definition of FCB-equivalence is abstract and hardly of any use when synthesizing structured nets from unstructured ones. Accordingly, we employ a more convenient way of reasoning about FCB-equivalence based on the ordering relations of occurrence nets. The idea is that any pair of nodes in an occurrence net can be in a precedence, conflict, or concurrent ordering relation as defined below, and these ordering relations can be used to reason about FCB-equivalence. 
Definition 13 (Ordering relations).

Let $N=(P, T, F)$ be an occurrence net and let $x, y \in X$ be two nodes of $N$.

$\circ x$ precedes $y$, denoted by $x \sim_{N} y$, iff $(x, y) \in F^{+}$.

$\circ x$ and $y$ are in conflict, denoted by $x \#_{N} y$, iff $\exists t_{1}, t_{2} \in T, t_{1} \neq t_{2}:\left(\bullet t_{1} \cap \bullet t_{2} \neq\right.$ $\varnothing) \wedge t_{1} \sim_{N} x \wedge t_{2} \sim_{N} y$.

$\circ x$ and $y$ are concurrent, denoted by $x \|_{N} y$, iff they are neither in precedence, nor in conflict.

The set $\mathcal{R}=\left\{\sim_{N}, \#_{N}, \|_{N}\right\}$ forms the ordering relations of $N$.

Let $N=(P, T, F, \mathcal{T}, \lambda)$ be a labeled occurrence net and let $T^{\prime}=\{t \in T \mid \lambda(t) \neq \tau\}$. The $\lambda$-ordering relations of $N$ are formed by the set $\mathcal{R}_{\lambda}=\left\{\sim_{N} \cap T^{\prime} \times T^{\prime}\right.$, $\#_{N} \cap$ $\left.T^{\prime} \times T^{\prime}, \|_{N} \cap T^{\prime} \times T^{\prime}\right\}$. We say that two ordering relations are isomorphic if for each pair of observable transitions the ordering relation coincides.

\section{Definition 14 (Isomorphism of ordering relations).}

Let $N_{1}=\left(P_{1}, T_{1}, F_{1}, \mathcal{T}_{1}, \lambda_{1}\right)$ and $N_{2}=\left(P_{2}, T_{2}, F_{2}, \mathcal{T}_{2}, \lambda_{2}\right)$ be two labeled occurrence nets with distinctive labelings. Let $T_{1}^{\prime}$ and $T_{2}^{\prime}$ denote non- $\tau$ transitions of $N_{1}$ and $N_{2}$, respectively. Two $\lambda$-ordering relations $\mathcal{R}_{\lambda_{1}}$ of $N_{1}$ and $\mathcal{R}_{\lambda_{2}}$ of $N_{2}$ are isomorphic, denoted by $\mathcal{R}_{\lambda_{1}} \cong \mathcal{R}_{\lambda_{2}}$, iff there is a bijection $\gamma: T_{1}^{\prime} \rightarrow T_{2}^{\prime}$, such that:

$\circ \forall t \in T_{1}^{\prime}: \lambda_{1}(t)=\lambda_{2}(\gamma(t))$, and

$\circ \forall t_{1}, t_{2} \in T_{1}^{\prime}:\left(t_{1} \sim_{N_{1}} t_{2} \wedge \gamma\left(t_{1}\right) \leadsto N_{2} \gamma\left(t_{2}\right)\right) \vee\left(t_{2} \sim_{N_{1}} t_{1} \wedge \gamma\left(t_{2}\right) \sim_{N_{2}} \gamma\left(t_{1}\right)\right) \vee$ $\left(t_{1} \#_{N_{1}} t_{2} \wedge \gamma\left(t_{1}\right) \#_{N_{2}} \gamma\left(t_{2}\right)\right) \vee\left(t_{1}\left\|_{N_{1}} t_{2} \wedge \gamma\left(t_{1}\right)\right\|_{N_{2}} \gamma\left(t_{2}\right)\right)$.

Finally, we show that two occurrence nets with isomorphic ordering relations are FCB-equivalent, and vice-versa. This result is exploited in the next section.

Theorem 1. Let $S_{1}=\left(N_{1}, M_{i}^{1}\right), N_{1}=\left(P_{1}, T_{1}, F_{1}, \mathcal{T}_{1}, \lambda_{1}\right)$, and $S_{2}=\left(N_{2}, M_{i}^{2}\right)$, $N_{2}=\left(P_{2}, T_{2}, F_{2}, \mathcal{T}_{2}, \lambda_{2}\right)$, be two labeled occurrence systems with distinctive labelings and $T_{1}^{\prime} \subseteq T_{1}, T_{2}^{\prime} \subseteq T_{2}$ observable transitions, such that there exists bijection $\psi: T_{1}^{\prime} \rightarrow T_{2}^{\prime}$ for which holds $\lambda_{1}(t)=\lambda_{2}(\psi(t))$, for all $t \in T_{1}^{\prime}$. Let $\mathcal{R}_{\lambda_{1}}$ and $\mathcal{R}_{\lambda_{2}}$ be the $\lambda$-ordering relations of $N_{1}$ and $N_{2}$. Then, it holds:

$$
S_{1} \approx S_{2} \Leftrightarrow \mathcal{R}_{\lambda_{1}} \cong \mathcal{R}_{\lambda_{2}}
$$

Proof. We prove each direction of the equality separately.

$(\Rightarrow)$ Let $S_{1}$ and $S_{2}$ be FCB-equivalent. We want to show that $\mathcal{R}_{\lambda_{1}} \cong \mathcal{R}_{\lambda_{2}}$.

Let us assume that $S_{1} \approx S_{2}$ holds, but $\mathcal{R}_{\lambda_{1}} \cong \mathcal{R}_{\lambda_{2}}$ does not hold. Furthermore, let us consider transitions $t_{i}^{1}, t_{j}^{1} \in T_{1}^{\prime}$ that are in one-to-one correspondence with transitions $t_{i}^{2}, t_{j}^{2} \in T_{2}^{\prime}$, i.e., $\lambda_{1}\left(t_{i}^{1}\right)=\lambda_{2}\left(\psi\left(t_{i}^{2}\right)\right)$ and $\lambda_{1}\left(t_{j}^{1}\right)=\lambda_{2}\left(\psi\left(t_{j}^{2}\right)\right)$. All scenarios can be reduced to the following two cases:

Case 1: $\left(t_{i}^{1} \|_{N_{1}} t_{j}^{1}\right.$ or $t_{i}^{1} \sim_{N_{1}} t_{j}^{1}$, and $\left.t_{i}^{2} \#_{N_{2}} t_{j}^{2}\right)$. If $t_{i}^{1} \|_{N_{1}} t_{j}^{1}$ or $t_{i}^{1} \sim_{N_{1}} t_{j}^{1}$, then there exists process $\pi_{1}$ in $S_{1}$ that contains $t_{i}^{1}$ and $t_{j}^{1}$. If $t_{i}^{2} \#_{N_{2}} t_{j}^{2}$, then there exists no process $\pi_{2}$ in $S_{2}$ that contains $t_{i}^{2}$ and $t_{j}^{2}$.

Case 2: $\left(t_{i}^{1} \gg_{N_{1}} t_{j}^{1}\right.$, and $t_{j}^{2} \sim_{N_{2}} t_{i}^{2}$ or $\left.t_{i}^{2} \|_{N_{2}} t_{j}^{2}\right)$. Let $\pi_{1}$ be a process in $S_{1}$ that contains $t_{i}^{1}$ and $t_{j}^{1}$, and let $\pi_{2}$ be a process in $S_{2}$ that contains $t_{i}^{2}$ and $t_{j}^{2}$. Then, there exists no $\phi \subseteq \psi$, such that $\phi$ is an order-isomorphism between $\lambda$-abstractions of $\pi_{1}$ and $\pi_{2}$.

In both cases we reach the contradiction, i.e., systems $S 1$ and $S 2$ cannot be FCB-equivalent if the $\lambda$-ordering relations are not isomorphic. 
$(\Leftarrow)$ Let $\mathcal{R}_{\lambda_{1}} \cong \mathcal{R}_{\lambda_{2}}$. We want to show that $S_{1}$ and $S_{2}$ are FCB-equivalent.

Let us assume that $\mathcal{R}_{\lambda_{1}} \cong \mathcal{R}_{\lambda_{2}}$ holds, but $S_{1} \approx S_{2}$ does not hold. Then, for instance, in $S_{1}$ there exists process $\pi_{1}^{\prime}$ that has no corresponding orderisomorphic process in $S_{2}$. Suppose that $\pi_{1}^{\prime}$ has the minimal size among all such processes, i.e., any prefix of $\pi_{1}^{\prime}$ has a corresponding order-isomorphic process in $S_{2}$. Let $\pi_{1}^{\prime}$ be an extension of $\pi_{1}$ by exactly one observable transition $t_{j}^{1} \in T_{1}^{\prime}$. Let $\pi_{2}$ be a process in $S_{2}$ that is order-isomorphic with $\pi_{1}$. Let $t_{j}^{2} \in T_{2}^{\prime}$ be in one-to-one correspondence with $t_{j}^{1}$, i.e., $\lambda_{1}\left(t_{j}^{1}\right)=\lambda_{2}\left(\psi\left(t_{j}^{2}\right)\right)$. All scenarios can be reduced to the following three cases:

Case 1: There exists process $\pi_{2}^{\prime}$ that contains $t_{j}^{2}$ and is an extension of $\pi_{2}$ by one observable transition. Moreover, there exists $t_{i}^{1} \in T_{1}^{\prime}$ in $\pi_{1}$, such that $t_{i}^{1} \sim_{N_{1}} t_{j}^{1}$. However, it holds $t_{i}^{2} \|_{N_{2}} t_{j}^{2}$, for $t_{i}^{2} \in T_{2}^{\prime}$, such that $\lambda_{1}\left(t_{i}^{1}\right)=\lambda_{2}\left(\psi\left(t_{i}^{2}\right)\right)$; otherwise there exists an order-isomorphism $\phi \subseteq \psi$ between $\pi_{1}^{\prime}$ and $\pi_{2}^{\prime}$.

Case 2: There exists no process $\pi_{2}^{\prime}$ that contains $t_{j}^{2}$ and is an extension of $\pi_{2}$. Moreover, there exists $t_{i}^{1} \in T_{1}^{\prime}$ in $\pi_{1}$, such that $t_{i}^{1} \leadsto N_{1} t_{j}^{1}$. However, it holds $t_{i}^{2} \#_{N_{2}} t_{j}^{2}$, for $t_{i}^{2} \in T_{2}^{\prime}$, such that $\lambda_{1}\left(t_{i}^{1}\right)=\lambda_{2}\left(\psi\left(t_{i}^{2}\right)\right)$.

Case 3: There exists process $\pi_{2}^{\prime}$ that contains $t_{j}^{2}$ and is an extension of $\pi_{2}$, but not by only one observable transition. Then, there exists $t_{k}^{2} \in T_{2}^{\prime}$ and process $\pi_{2}^{\prime \prime}$ in $S_{2}$, such that $t_{k}^{2} \sim_{N_{2}} t_{j}^{2}, \pi_{2}^{\prime \prime}$ is prefix of $\pi_{2}^{\prime}$, and $\pi_{2}$ is prefix of $\pi_{2}^{\prime \prime}$. However, $t_{k}^{1} \in T_{1}^{\prime}, \lambda_{1}\left(t_{k}^{1}\right)=\lambda_{2}\left(\psi\left(t_{k}^{2}\right)\right)$, is not in $\pi_{1}^{\prime}$ and, hence, $t_{k}^{1} \rtimes_{N_{1}} t_{j}^{1}$. In all three cases we reach the contradiction, i.e., the $\lambda$-ordering relations cannot be isomorphic if systems $S_{1}$ and $S_{2}$ are not FCB-equivalent.

\section{Synthesis of Structured Process Models}

The key idea of the proposed structuring method is to compute the ordering relations of every rigid component, and to synthesize a structured process component from these ordering relations (if such a structured process component exists). A structured process component is one whose RPST contains only trivials, bonds and polygons. Accordingly, what we need is to find such structures in the graph induced by the ordering relations of the component. To this end, we rely on the concept of modular decomposition [19]. Below we discuss how to compute the ordering relations of a process component and then we use the output of this step to synthesize a structured component based on the modular decomposition.

\subsection{Computing Ordering Relations}

In order to compute the ordering relations of tasks in a process component, we first need to build a corresponding occurrence net, using a procedure known as unfolding [20]. For example, Fig.5 presents the occurrence net for the rigid component $R 1$ in Fig.3. The occurrence net may include multiple transitions referring to the same task, e.g., transitions $t_{c, 1}$ and $t_{c, 2}$ refer to task $c$. If we used the ordering relations computed from the occurrence net to synthesize a structured process component, the component would contain many duplicate tasks. Fortunately, for any safe net there exists a prefix of its occurrence net, called the complete prefix unfolding [20], that is more compact than the occurrence net 
but contains all the information about markings contained in the occurrence net. Moreover, this prefix is finite (even for safe nets with cycles). The complete prefix unfolding is obtained by truncating the occurrence net in points where the information about reachable markings starts to be redundant.

\section{Definition 15 (Complete Prefix Unfolding, Cutoff transition).}

Let $N=(P, T, F)$ be an occurrence net.

- A local configuration $\lceil t\rceil$ of a transition $t$ in an occurrence net is the set of transitions that precede $t$, i.e., $[t]=\left\{t^{\prime} \in T \mid\left(t^{\prime}, t\right) \in F^{*}\right\}$.

- The final marking of a local configuration Mark $([t])$ is the set of places that are marked after all the transitions in $\lceil t\rceil$ fire.

- An adequate order $\triangleleft$ is a strict well-founded partial order on local configurations, so that $\lceil t\rceil \subset\left\lceil t^{\prime}\right\rceil$ implies $\lceil t\rceil \triangleleft\left\lceil t^{\prime}\right\rceil^{3}$.

- A transition $t$ of an occurrence net is a cutoff transition if there exists a corresponding transition $t^{\prime}$, such that $\operatorname{Mark}(\lceil t\rceil)=\operatorname{Mark}\left(\left\lceil t^{\prime}\right\rceil\right)$ and $\left\lceil t^{\prime}\right\rceil \triangleleft\lceil t\rceil$.

- A complete prefix unfolding is the greatest backward closed subnet of an occurrence net containing no transitions after cutoff transitions.

The dotted lines in Fig.5 indicate which parts of the occurrence net are truncated in the complete prefix unfolding. In the unfolding, transition $t_{v}$ is a cutoff transition.

Alg.1 (adapted from [21]) computes the ordering relations based on a complete prefix unfolding. This algorithm has a low polynomial time to the size of the net. However, the overall complexity of computing ordering

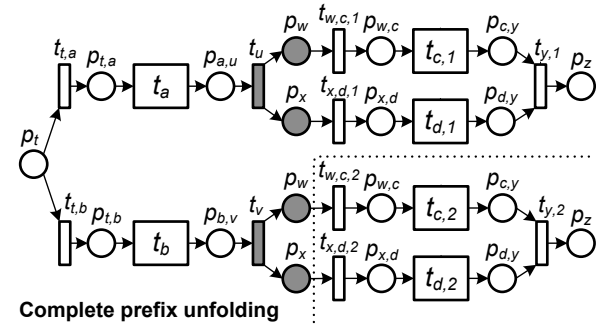

Fig. 5. Occurrence net and complete prefix unfolding of the running example relations is dominated by the exponential worst-case complexity of computing the prefix unfolding, which is an NP-complete problem. Observe that in the case of and rigids this step is not required as the corresponding WF-net is always an occurrence net. Besides, we do not compute the prefix unfolding over the whole net, but only on individual rigid components of the net. Tests we have conducted with sample process models show that the prefix unfolding computation takes sub-second times ${ }^{4}$. This finding is in line with other work that have empirically shown that prefix unfolding computation is efficient in practice [20].

Alg.1 comprises two phases. First, it computes the ordering relations of transitions on the unfolding according to Definition 13. Then, it updates the relations of transitions in the local configuration of every cutoff transition to overcome the effects of truncation. The update must be performed in reverse topological order. For instance, the algorithm will assert $\left(t_{b} \leadsto t_{c, 1}\right)$ in addition to $\left(t_{a} \leadsto t_{c, 1}\right)$, i.e., task $c$ may be preceded by either $a$ or $b$. Note that we impose an additional requirement

\footnotetext{
${ }^{3}$ Several definitions of adequate order exist; we use the one defined in [20], because it has been shown to generate compact unfoldings.

${ }^{4}$ Using the Mole tool for prefix unfolding http://www.fmi.uni-stuttgart.de/szs/ tools/mole/ which implements the algorithm in [20].
} 


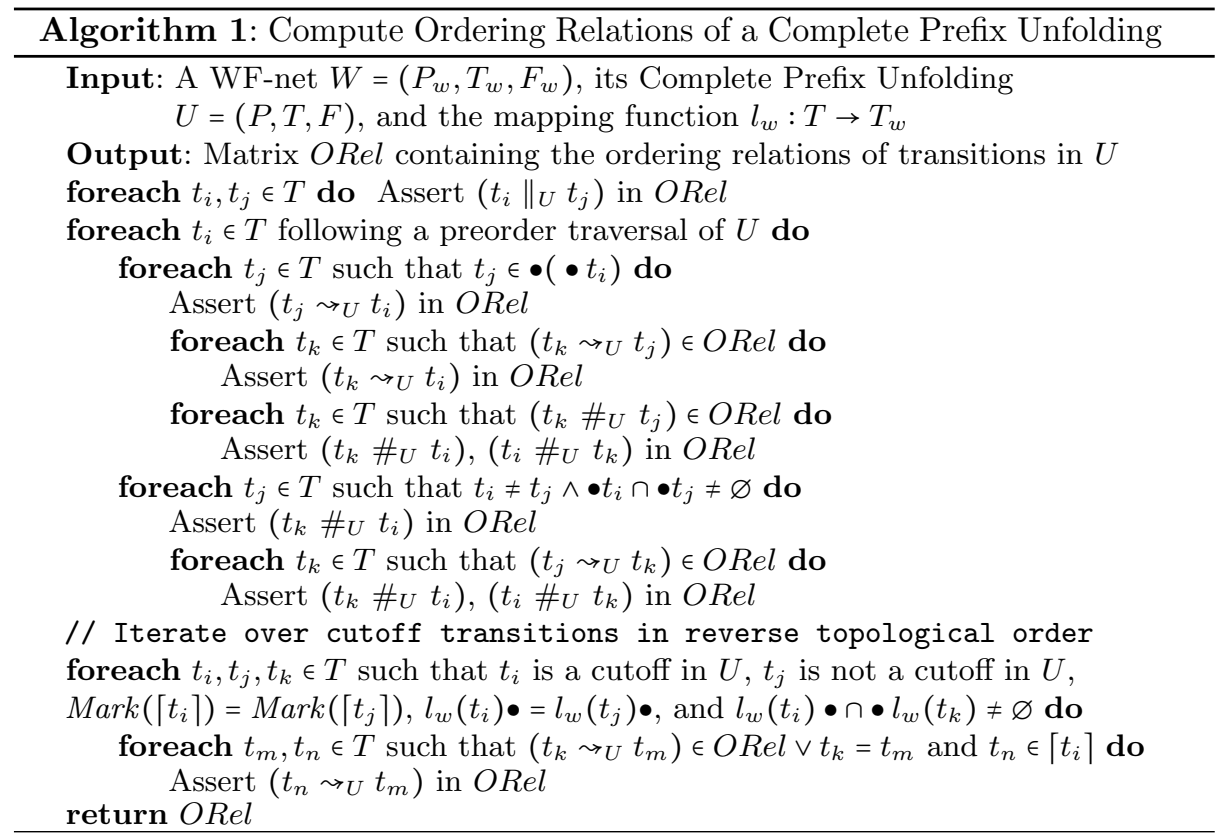

i.e., postsets of a cutoff transition and its corresponding transition must map to the same set of places in the WF-net, for all cutoff transitions. If a complete prefix unfolding does not meet this requirement, it must be expanded.

\subsection{From Ordering Relations to Process Models}

This section presents the algorithm for synthesizing a well-structured process model that is fully concurrent bisimilar with a given (unstructured) model. Also, we identify the cases when an equivalent well-structured model does not exist.

According to Theorem 1, two process models are fully concurrent bisimilar, iff they demonstrate same ordering relations. Given an (unstructured) process model, the algorithm proceeds by computing its ordering relations, as discussed in Sect. 5.1. Afterwards, the algorithm attempts to synthesize a well-structured model with the same ordering relations.

Let $N=(P, T, F, \mathcal{T}, \lambda)$ be a labeled occurrence net. The ordering relations graph of $N$ is a triple $\mathcal{G}_{\lambda}=(V, E, \mathcal{L})$, where $V$ is the set of non- $\tau$ transitions of $N, \mathcal{L}=\{\epsilon, \leadsto, \#, \|\}$ is a set of labels, and $E: V \times V \rightarrow \mathcal{L}$ is an edge labeling function, such that $E(x, y)=\oplus, x, y \in V$ and $\oplus \in \mathcal{L} \backslash \epsilon$, if $x \oplus_{N} y$, otherwise $E(x, y)=\epsilon$. Self-relations are ignored, i.e., $E(x, x)=\epsilon, x \in V$. Observe that $\mathcal{G}_{\lambda}$ is an alternative representation of $\lambda$-ordering relations $\mathcal{R}_{\lambda}$ of $N$.

Fig.6(a) shows the ordering relations graph of a complete prefix unfolding that is given in Fig.5. As the conflict and concurrency relations are symmetric, the corresponding edges are visualized as two-sided arrows; solid and dotted for the conflict $(a \# b)$ and concurrency $(c \| d)$ relation, respectively. Regular arrows reflect the precedence relation, which is transitive and asymmetric. Edges that have $\epsilon$ labels are not visualized. Because of the precedence relation, most of the ordering relations graphs are asymmetric. 


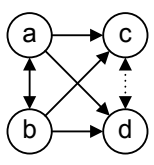

(a)

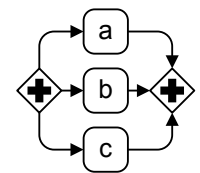

(b)

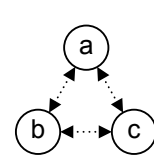

(c)

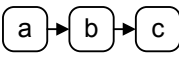

(d)

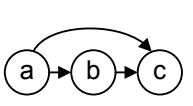

(e)

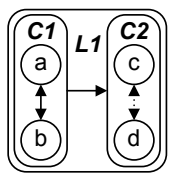

(f)

Fig. 6. (a) An ordering relations graph, (b) an and bond component, (c) an and complete module, (d) a polygon component, (e) a linear module, (f) the MDT of (a)

The RPST of a well-structured model is composed of trivial, polygon, and bond (either and or xor) components. Contrary to a rigid component that can have an arbitrary topology, the structure of each component of a well-structured model is well-defined and has a precise structural characterization in terms of the corresponding ordering relations graph. The ordering relations graph of a bond is a complete graph, or a clique. All edges in the graph have the same label: \# for xor bonds and $\|$ for and bonds. This topology is consistent with the intuition behind: all nodes in a xor bond are in conflict, i.e., only one is executed; all nodes in an and bond are concurrently executed. Fig.6(b) shows an and bond with three parallel branches, whereas Fig.6(c) shows the corresponding clique of concurrent relations. In the cases of a trivial and polygon component, the ordering relations graph is a direct acyclic graph representing the transitive closure, or the total order, of the precedence relation. All edges of the graph are labeled $\leadsto$. Fig.6(d) shows a polygon composed of three activities, whereas Fig.6(e) presents the corresponding transitive closure over the precedence relation.

Let $\mathcal{G}=(V, E, \mathcal{L})$ be an ordering relations graph. A module $M \subseteq V$ of $\mathcal{G}$ is a non-empty subset of transitions that have a uniform relations with transitions $V \backslash M$, i.e., $\forall x, y \in M \forall z \in V \backslash M: E(x, z)=E(y, z) \wedge E(z, x)=E(z, y)$. Note that singleton sets of $V$ are referred to as trivial modules.

Definition 16 (Complete, Linear, Primitive).

Let $M$ be a non-singleton module of $\mathcal{G}$.

○ $M$ is complete $(C)$, iff $\exists l \in\{\#, \|\} \forall x, y \in M, x \neq y: E(x, y)=l$, i.e., the subgraph induced by $M$ is a complete graph, or a clique. If $l=\#$, then $M$ is xor complete, otherwise $M$ is and complete.

- $M$ is linear $(L)$, iff there exists a linear order $\left(x_{1}, \ldots, x_{\left|T^{\prime}\right|}\right)$ of elements of $T^{\prime}$, such that $E\left(x_{i}, x_{j}\right)=\leadsto$, if $i<j$, and $E\left(x_{i}, x_{j}\right)=\epsilon$ otherwise.

- If $M$ is neither complete, nor linear, then $M$ is primitive $(P)$.

The following proposition summarizes relations between components of a process model and modules of an ordering relations graph.

Proposition 1. Let $C_{1}$ be a process component and let $M_{1}$ be the corresponding ordering relations graph. Let $M_{2}$ be an ordering relations graph and let $C_{2}$ be the corresponding process component.

1. If $C_{1}$ is trivial or polygon, then $M_{1}$ is linear.

2. If $M_{2}$ is linear, then there exists $C_{2}$ that is trivial or polygon.

3. If $C_{1}$ is and (xor) bond, then $M_{1}$ is and (xor) complete.

4. If $M_{2}$ is and (xor) complete, then there exists $C_{2}$ that is and (xor) bond.

Two modules $M_{1}$ and $M_{2}$ of $\mathcal{G}$ overlap, iff they intersect and neither is a subsetof 
the other, i.e., $M_{1} \backslash M_{2}, M_{1} \cap M_{2}$, and $M_{2} \backslash M_{1}$ are all non-empty. $M_{1}$ is strong, iff there exists no module $M_{2}$ of $\mathcal{G}$, such that $M_{1}$ and $M_{2}$ overlap. The modular decomposition substitutes each strong module of a graph by a new vertex and proceeds recursively. The result is a rooted, unique tree called the Modular Decomposition Tree, which can be computed in linear time [19].

Definition 17 (Modular Decomposition Tree). Let $\mathcal{G}=(V, E, \mathcal{L})$ be an ordering relations graph. The Modular Decomposition Tree (MDT) of $\mathcal{G}$, denoted by $\operatorname{MDT}(\mathcal{G})$, is a containment hierarchy of all strong modules of $\mathcal{G}$.

Fig.6(f) shows the MDT of the ordering relations graph that is proposed in Fig.6(a). Each module is enclosed in a box with rounded corners. Note that module names hint at their class. For instance, module $C 1$ is a complete module, and is composed of two nodes $a$ and $b$ that are in conflict relation, $a \# b$. Therefore, $C 1$ is a xor complete module. Similarly, $C 2$ is an and complete module. By treating both modules as singletons, the modular decomposition identifies that they are in total order and, hence, form a linear module $L 1$.

We are now ready to present the main result of this section.

Theorem 2. Let $\mathcal{G}$ be an ordering relations graph. The Modular Decomposition Tree of $\mathcal{G}$ has no primitive module, iff there exists a well-structured process model $W$ such that $\mathcal{G}$ is the ordering relations graph of $W$.

Proof. Let $\mathcal{G}=(V, E, \mathcal{L})$ be an ordering relations graph.

$(\Rightarrow)$ Assume that the MDT of $\mathcal{G}$ has no primitive module. We show now by structural induction on the MDT of $\mathcal{G}$ that there exists a well-structured process model $W$ with ordering relations $\mathcal{G}$. The MDT of $\mathcal{G}$ contains singleton, linear, and complete, and xor complete modules.

Base: If the MDT of $\mathcal{G}$ consists of a single module $M$, then $M$ is singleton and $W$ is a process model composed of a single task $m \in M$.

Step: Let $M$ be a module of the MDT of $\mathcal{G}$ such that each child module of $M$ has a corresponding well-structured process model. If $M$ is linear, then $W$ can be a trivial or polygon component composed from children of $M$, cf., 2 in Prop. 1. If $M$ is complete, then $W$ can be a bond component, either and or xor, composed from children of $M$, cf., 4 in Prop. 1. In both cases, $M$ has a corresponding well-structured process model.

Therefore, there exists a well-structured process model $W$ composed from children of module $V$ of $\mathcal{G}$ that has ordering relations graph $\mathcal{G}$.

$(\Leftarrow)$ Let $W$ be a well-structured process model with ordering relations graph $\mathcal{G}$. We want to show that the MDT of $\mathcal{G}$ has no primitive module. Because $W$ is well-structured, the RPST of $W$ has no rigid component. The corresponding ordering relations graph of a non-rigid component, i.e., trivial, polygon, or bond component, is either complete or linear, cf., 1 and 3 in Prop. 1. If $W$ is composed of a single task, then $\mathcal{G}$ consists of one singleton trivial module.

Finally, we detail the approach for structuring acyclic rigid components in Alg.2.

Based on all previous results, Alg.2 synthesizes, whenever possible, the RPST of an FCB-equivalent well-structured component for a given acyclic rigid component. No variables are introduced. Task duplication depends on the "quality" of the prefix unfolding. The complexity of the algorithm is determined by the exponential 


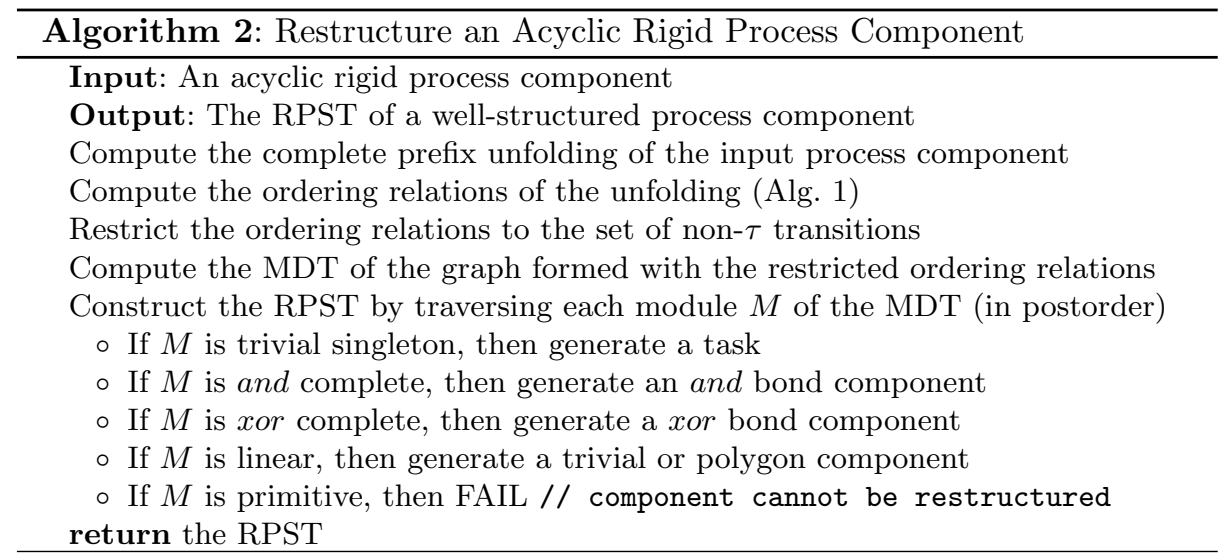

complexity of the unfolding (see earlier discussion). All other steps are polynomial. In the case of an and rigid, the unfolding is not needed because the WF-net of an and rigid is already an occurrence net. The algorithm fails if the input process component is inherently unstructured, such as the process component in Fig.7(a). In this particular case, the ordering relations graph forms a single primitive module, cf., Fig.7(b). Note that the unfolding step duplicates task $f$.

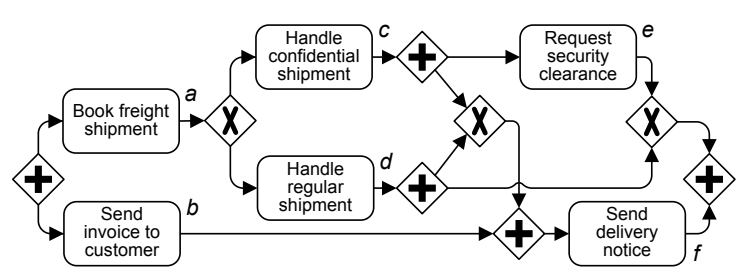

(a)

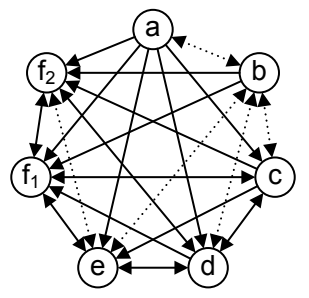

(b)

Fig. 7. (a) A rigid process component and (b) its ordering relations graph

\section{Conclusion}

We conclude that a sound and safe acyclic process model is inherently unstructured if its RPST has a rigid component for which the modular decomposition of its ordering relations contains a primitive. In all other cases, Algorithm 2 applied to each rigid in the RPST constructs an equivalent structured model. We have thus provided a characterization of the class of structured acyclic process models under FCB equivalence, and a complete structuring method. This method is implemented in a tool, namely bpstruct, that structures BPMN models exported from Oryx ${ }^{5}$. The tool is available at https://code.google.com/p/bpstruct/.

This method can also be used to structure models with SESE cycles, even if these cycles contain unstructured components. In this case, the unstructured components and the cycles are in different nodes of the RPST. However, the

\footnotetext{
${ }^{5}$ http://oryx-project.org/
} 
proposed method cannot deal with models with arbitrary cycles. Also, the results do not apply to models with OR-joins, complex gateways, exception handlers and non-interrupting events. Future work will aim at lifting these restrictions.

Acknowledgments. This research is partly funded by the ERDF via the Estonian Center of Excellence in Computer Science.

\section{References}

1. Kiepuszewski, B., ter Hofstede, A.H.M., Bussler, C.: On Structured Workflow Modelling. In: CAiSE. Volume 1789 of LNCS. (2000) 431-445

2. Laue, R., Mendling, J.: The Impact of Structuredness on Error Probability of Process Models. In: UNISCON. Volume 5 of LNBIP. (2008) 585-590

3. Laguna, M., Marklund, J.: Business Process Modeling, Simulation, and Design. Prentice Hall (2005)

4. Combi, C., Posenato, R.: Controllability in Temporal Conceptual Workflow Schemata. In: BPM. Volume 5701 of LNCS. (2009) 64-79

5. Oulsnam, G.: Unravelling unstructured programs. Comput. J. 25 (1982) 379-387

6. Liu, R., Kumar, A.: An Analysis and Taxonomy of Unstructured Workflows. In: BPM. Volume 3649 of LNCS. (2005) 268-284

7. Hauser, R., Friess, M., Küster, J.M., Vanhatalo, J.: An Incremental Approach to the Analysis and Transformation of Workflows Using Region Trees. IEEE Transactions on Systems, Man, and Cybernetics, Part C 38 (2008) 347-359

8. Polyvyanyy, A., García-Bañuelos, L., Weske, M.: Unveiling Hidden Unstructured Regions in Process Models. In: OTM. Volume 5870 of LNCS. (2009) 340-356

9. Hauser, R., Koehler, J.: Compiling Process Graphs into Executable Code. In: GPCE. Volume 3286 of LNCS. (2004) 317-336

10. Koehler, J., Hauser, R.: Untangling Unstructured Cyclic Flows - A Solution Based on Continuations. In: OTM. Volume 3290 of LNCS. (2004) 121-138

11. Ouyang, C., Dumas, M., van der Aalst, W.M.P., ter Hofstede, A.H.M., Mendling, J.: From business process models to process-oriented software systems. ACM Trans. Softw. Eng. Methodol. 19 (2009)

12. Kiepuszewski, B., ter Hofstede, A.H.M., van der Aalst, W.M.P.: Fundamentals of Control Flow in Workflows. Acta Inf. 39 (2003) 143-209

13. Vanhatalo, J., Völzer, H., Koehler, J.: The Refined Process Structure Tree. Data \& Knowledge Engineering 68 (2009) 793-818

14. Polyvyanyy, A., Vanhatalo, J., Völzer, H.: Simplified computation and generalization of the refined process structure tree. Technical Report RZ 3745, IBM (2009)

15. van der Aalst, W.M.P.: Verification of Workflow Nets. In: Application and Theory of Petri Nets. Volume 1248 of LNCS. (1997) 407-426

16. van der Aalst, W.M.P.: Workflow verification: Finding control-flow errors using petri-net-based techniques. In: BPM. Volume 1806 of LNCS. (2000) 161-183

17. van Glabbeek, R.J.: The Linear Time-Branching Time Spectrum (Extended Abstract). In: CONCUR. Volume 458 of LNCS. (1990) 278-297

18. Best, E., Devillers, R.R., Kiehn, A., Pomello, L.: Concurrent bisimulations in petri nets. Acta Inf. 28 (1991) 231-264

19. McConnell, R.M., de Montgolfier, F.: Linear-time modular decomposition of directed graphs. Discrete Applied Mathematics 145 (2005) 198-209

20. Esparza, J., Römer, S., Vogler, W.: An Improvement of McMillan's Unfolding Algorithm. FMSD 20 (2002) 285-310

21. Kondratyev, A., Kishinevsky, M., Taubin, A., Ten, S.: Analysis of Petri Nets by Ordering Relations in Reduced Unfoldings. FMSD 12 (1998) 5-38 


\section{University Library}

\section{- M M N E R VA A gateway to Melbourne's research publications}

Minerva Access is the Institutional Repository of The University of Melbourne

Author/s:

Polyvyanyy, A;Garcia-Banuelos, L;Dumas, M

Title:

Structuring Acyclic Process Models

Date:

2010-01-01

Citation:

Polyvyanyy, A., Garcia-Banuelos, L. \& Dumas, M. (2010). Structuring Acyclic

Process Models. Hull, R (Ed.) Mendling, J (Ed.) Tai, S (Ed.) BUSINESS PROCESS

MANAGEMENT, 6336, pp.276-+. SPRINGER-VERLAG BERLIN. https://

doi.org/10.1007/978-3-642-15618-2_20.

Persistent Link:

http://hdl.handle.net/11343/241604 